\title{
Reliability Systems and Optimal Control - Goal Realization Probability
}

\author{
Aleksandra Rajcevic \\ Faculty of Civil Engineering, University of Belgrade, Belgrade, Serbia
}

Email address:

aleksandra_rt@mts.rs

\section{To cite this article:}

Aleksandra Rajcevic. Reliability Systems and Optimal Control - Goal Realization Probability. American Journal of Applied Mathematics. Vol. 8, No. 6, 2020, pp. 293-299. doi: 10.11648/j.ajam.20200806.11

Received: October 14, 2020; Accepted: October 29, 2020; Published: November 11, 2020

\begin{abstract}
In this theoretical article is considered reliability cybernetics systems with probability theory. Optimal control and managing assigned goals of system are considered after control actions were assigned with a determined probability of realization. Real system is set of elements functionally connected in one whole for achieving determined goal using, transforming and exchanging energy, resources and informations. Real system mainly is presented as functional and physical whole. System researched by cybernetics could be school, metallic company or concrete factory. New concepts are involved in researching cybernetics systems reliability systems and optimal control. Controlling system is an action on object of control which optimized functioning of that object. Optimal control is substituted set of control actions which are got favorable optimal criterion value. Optimal criterions are considered as values of gains or value of losses. Reliability systems is property of system to realize his function or goal with some probability, having in mind reliability all of system elements. Realization wanted output or goal realization is calculated as intersection projected system parts realizations with determined probability. In this paper will be shown three different systems regarded to how they executed their controlling actions. Probability of realization system goals are calculated for two cases: maximum and minimum reliability. Intention is to show and calculate reliability systems with high human resources representation.
\end{abstract}

Keywords: Probability, Cybernetics, Reliability Systems, Controlling

\section{Introduction}

Cybernetics is field of science which analyzed methods and procedures of controlling systems and researched controllable systems. Cybernetics was applied in researching different systems which could be:

technical

biological

organizational.

Living areas where cybernetics was applied are social, business, water resource systems in which man was taken part and have had effort for reaching optimal goals.

Real systems is set of elements (units, components, factors, parts, sections) functionally connected in one whole for achieving determined goal using, transforming and exchanging energy, resources and (or) informations. Real system mainly is presented as functional and physical whole. Examples of cybernetics system are simple: functioning of school, metallic company or concrete factory, they are all organizational or technical systems researched by cybernetics. [1]

Researched system is functioned in surroundings and influence of environment is established through vector uncertainty - accident vector v. Most regular systems which are met in human society could describe schematically and analyzed by cybernetics.

Every cybernetics system is described with input and output values. System output value is the output action of system on environment in which changed values were interested, on intention to reach certain goal, which was presented as result of dynamical system behavior. Output value is marked with $y(t)$.

Input values which are acted on system could be divided on two kinds: control actions $u(t)$ and accident actions. Describing one composite system with $r$ inputs and $m$ outputs is:

$\left\{\mathrm{y}_{1}(\mathrm{t}), \mathrm{y}_{2}(\mathrm{t}), \ldots, \mathrm{y}_{\mathrm{m}}(\mathrm{t})\right\}=\{\mathrm{k}\}\left\{\mathrm{u}_{1}(\mathrm{t}), \mathrm{u}_{2}(\mathrm{t}), \ldots \mathrm{u}_{\mathrm{r}}(\mathrm{t})\right\}$

$\{\mathrm{y}\}=\{\mathrm{k}\}\{\mathrm{u}(\mathrm{t})\}$ 
Operator k characterized properties of analyzed system, transforming inputs in systems outputs.

Mathematical model of system is formal mathematical description of system (with symbols, operations and relations) which provided total analyses of his dynamic behavior for accidental input values changes and unintentional starting conditions. It is used to describe technical systems.

Mathematical model of systems could determined in a form of functional reliance input and output values or in a form of graphics. [1, 4-6]

In this paper will be shown three different systems regarded to how they are executed their control actions, analyzed their structure and calculated probability of their goals realization.

\section{Reliability Systems and Optimal Control}

Controlling system is action on object of control which optimized functioning of that object, chosen from set of possible actions on system based on available informations. In real systems most often control on object is realized with few control actions, which are limited with some boundary conditions.

$$
\begin{aligned}
& U-\text { controlling } \\
& \mathrm{u}=\left\{\mathrm{u}_{\mathrm{i}}, \mathrm{u}_{\mathrm{imin}} \leq \mathrm{u}_{\mathrm{i}} \leq \mathrm{u}_{\mathrm{imax}}, \mathrm{i}=1, \mathrm{r}\right\} \\
& \mathrm{y}=\mathrm{f}\left(\mathrm{y}_{\mathrm{d}}, \mathrm{u}, \mathrm{v}\right) \\
& \mathrm{v}-\text { accident vector }
\end{aligned}
$$

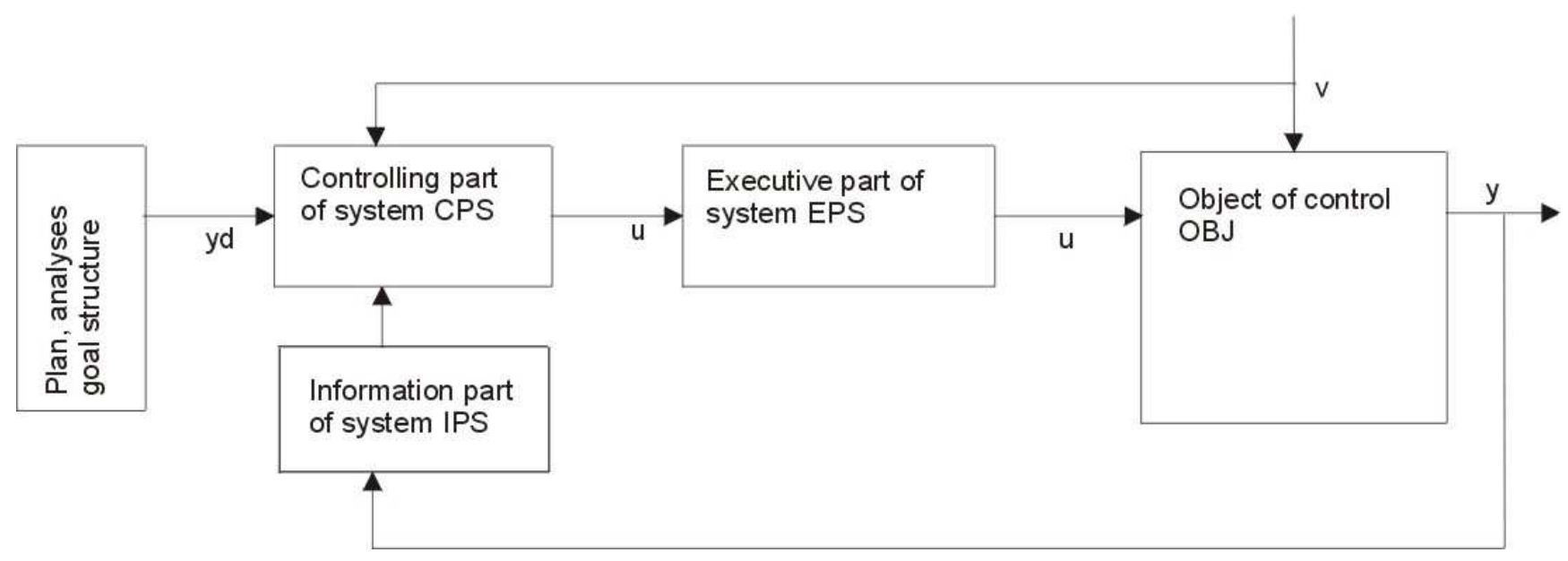

Figure 1. Structural scheme of controlled system - scheme 1 [2].

$\mathrm{y}_{\mathrm{d}}$ - demanded output

$\mathrm{y}$ - output value

$\mathrm{u}-$ system control

Controlling part of system (CPS) on scheme is assigned action or control $\mathrm{u}=\mathrm{u}\left(\mathrm{y}_{\mathrm{d}}, \mathrm{y}, \mathrm{v}\right)$ on control object, based on informations about system behavior. Executive part of system (ExPS) is executed control on object.

Object of control (OBJ - physical part of system) is presented physical or functional whole, where could find different representation of human resources, technique or machines. Most suitable and realistic object of control (factory, business company) is object with 50\% proportion of human labor and 50\% machine work. It will be shown through the calculation that this systems are achieved maximum reliability in functioning. On object of control is affected accident vector v. Information part of system (IPS) is got informations about system functioning.

Optimal control is substituted set of control actions which having determined limitations on system, are got most favorable optimal criterion value. When controlling is defined for each system, then limitations and optimal criterion also are defined. Optimal criterions are contemplated as values of gains or values of losses.

Reliability systems is property of system to realize his function or goal with some probability, having in mind reliability all of system elements. This way it is possible considered reliability functioning real and projected systems using probability theory. In next chapter are shown examples and calculations systems reliability.

\section{Calculations}

It will take into count three different systems: simple system, system with two executive parts and system with four executive parts, regarded to how they are executed their controlling instructions.

\subsection{Simple System}

On Scheme 1 is shown first considered system, simple system which could be school, textile factory, hotel or similar organizational system. Also it could be railway, subway or similar technical system.

Plan, analyze and goal structure part of system is analyzed several goals which could be opposed and is chosen and defined optimal control in form demanded output yd, which is determined system function realization. There could be more system goals and it could be demanded more complexes system outputs.

Informational part of system presented whole with controlling part of system. Informations about realized output 
of system is sent to the informational part of system (through system feedback loop). Based on this informations and informations about system accidents controlling part of system defined controlling or more control actions.

Realization wanted output or goal achievement yd is calculated as intersection of projected system parts realizations with determined probability.

$$
\begin{aligned}
& \mathrm{y}=\mathrm{u}\left(\mathrm{y}_{\mathrm{d}}, \mathrm{v}\right) \\
& \mathrm{v}-\mathrm{acciden} \text { vector, uncertainty evaluated as } 30 \% \\
& \mathrm{yd}-\text { demanded output } \\
& \mathrm{y}-\text { system output, controlling result } \\
& \mathrm{u}-\text { controlling action }
\end{aligned}
$$

Two cases are calculated, maximum and minimum reliability.

\subsubsection{Maximum System Reliability}

Estimated system elements reliability are high and realization probability is amount $\mathrm{p}=0.9$, event probability that system execute his function is counted as intersection single element realization events.

Information system is worked on two extreme possibilities - two hypotheses: is worked good with probability 0.95 and is worked with minimum success with probability 0.70 .

$$
\begin{aligned}
& \text { Pis }\left(\mathrm{p}_{\max }, \mathrm{p}_{\min }\right)=(0.95,0.70) \\
& \mathrm{P}(\mathrm{IPS})=1 / 2 * 0.95+1 / 2 * 0.70 \\
& \mathrm{P}(\mathrm{IPS})=0.825
\end{aligned}
$$

Information - controlling system work is presented as independent events union.

$$
\begin{gathered}
\mathrm{P}(\mathrm{A} \cup \mathrm{B})=\mathrm{P}(\mathrm{A})+\mathrm{P}(\mathrm{B})-\mathrm{P}(\mathrm{A}) * \mathrm{P}(\mathrm{B}) \\
\mathrm{P}(\mathrm{A})=\mathrm{P}(\mathrm{CPS})=0.9 \\
\mathrm{P}(\mathrm{B})=\mathrm{P}(\mathrm{IPS})=0.825 \\
\mathrm{P}(\mathrm{A} \cup \mathrm{B})=0.9+0.825-0.9 * 0.825 \\
\mathrm{P}(\mathrm{A} \cup \mathrm{B})=0.9825
\end{gathered}
$$

Plan and goal structure reliability is evaluated with probability $\mathrm{P}(\mathrm{GS})=1.0$

Reliability and work executive part of system is counted high with probability 0.9 .

$$
\mathrm{P}(\text { ExPS })=0.9
$$

Reliability and work controlling object (OBJ) is evaluated 0.7 . Accident vector is evaluated $v=0.3$.

$$
\mathrm{P}(\mathrm{OBJ})=0.7
$$

System probability in reaching demanded output or goal is:

$\mathrm{p}=\operatorname{CIPS}(\mathrm{p}=0.9825) \cap \operatorname{ExPS}(\mathrm{p}=0.9) \cap \mathrm{OBJ}(\mathrm{p}=0.7) \cap \mathrm{GS}(\mathrm{p}=1.0)$

$$
\begin{gathered}
y=0.9825 * 0.9 * 0.7 * 1.0 \mathrm{yd} \\
y=0.6190 \mathrm{yd}
\end{gathered}
$$

Maximum reliability system with high representation human resources (about 50\%) is counted on $\mathrm{y}=0.6190 \mathrm{yd}$.

\subsubsection{Minimum System Reliability}

As minimum reliability is counted goal reaching case with minimum reliability system elements, but with possibility for event realization. Hypotheses is that executive part of system and controlling part of system are worked with probability 0.5 , what is considered as one man decision.

$$
\mathrm{p}_{1}=\mathrm{p}_{2}=\mathrm{P}(\mathrm{CPS})=\mathrm{P}(\text { ExPS })=1 / 2=0.5 \text { (one man decision) }
$$

Information system reliability is shown:

$$
\begin{gathered}
\text { Pis }\left(\mathrm{p}_{\max }, \mathrm{p}_{\min }\right)=(0.95,0.70) \\
\mathrm{P}(\mathrm{IPS})=1 / 2 * 0.95+1 / 2 * 0.70 \\
\mathrm{P}(\mathrm{IPS})=0.825
\end{gathered}
$$

Information - controlling system work is presented as independent events union.

$$
\begin{gathered}
\mathrm{P}(\mathrm{A} \cup \mathrm{B})=\mathrm{P}(\mathrm{A})+\mathrm{P}(\mathrm{B})-\mathrm{P}(\mathrm{A}) * \mathrm{P}(\mathrm{B}) \\
\mathrm{P}(\mathrm{A})=\mathrm{P}(\mathrm{CPS})=0.5 \\
\mathrm{P}(\mathrm{B})=\mathrm{P}(\mathrm{IPS})=0.825 \\
\mathrm{P}(\mathrm{A} \cup \mathrm{B})=0.5+0.825-0.5 * 0.825 \\
\mathrm{P}(\mathrm{A} \cup \mathrm{B})=0.9125
\end{gathered}
$$

Plan and goal structure reliability is evaluated wit probability $\mathrm{P}(\mathrm{GS})=1.0$

Reliability and work controlling object (OBJ) is evaluated 0.7 . Accident vector is evaluated $\mathrm{v}=0.3$.

$$
\mathrm{P}(\mathrm{OBJ})=0.7
$$

System probability in reaching demanded output or goal is:

$$
\begin{gathered}
\mathrm{p}=\operatorname{CIPS}(\mathrm{p}=0.9125) \cap \operatorname{ExPS}(\mathrm{p}=0.5) \cap \mathrm{OBJ}(\mathrm{p}=0.7) \cap \mathrm{GS}(\mathrm{p}=1.0) \\
\mathrm{y}=0.9125 * 0.5 * 0.7 * 1.0=0.3194 \mathrm{yd}
\end{gathered}
$$

Realizing system goal with less reliable system elements is possible with probability $0.3194 \mathrm{yd}$.

\subsection{System with Two Executive Parts}

Plan and analyze part of system is defined goal structure and demanded output through goals optimization. [7]

Controlling part of system is defined controlling based on determined yd and information is got through feedback loops about system behavior $\mathrm{y}$ and accident $\mathrm{v}$.

Executive part of system have two executive parts which together are worked on control realization, having in mind that each could realize control action by his own.

Researched system is presented joined work of two executive parts, having the same control action $u$. 


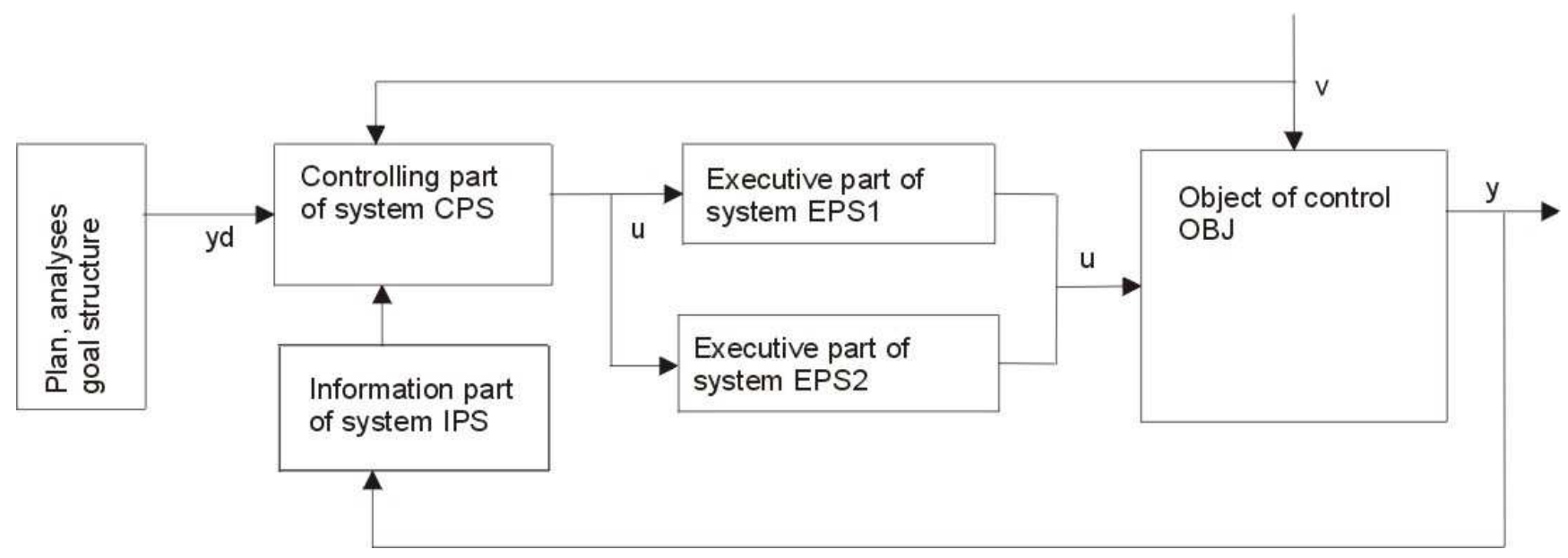

Figure 2. Structural scheme of controlled system - scheme 2 [2].

\subsubsection{Maximum System Reliability}

System reliability is counted with maximum elements reliability evaluated as realization probability 0.9. Executive part of system is taken into account as elements union (element executions are treated as independent events) and Law of total probability $[3,9,10]$ is applied for element probability calculation.

Information system reliability is shown:

$$
\begin{gathered}
\text { Pis }\left(p_{\max }, p_{\min }\right)=(0.95,0.70) \\
P(\text { IPS })=1 / 2 * 0.95+1 / 2 * 0.70 \\
P(I P S)=0.825
\end{gathered}
$$

Information - controlling system work is presented as independent events union.

$$
\begin{gathered}
\mathrm{P}(\mathrm{A} \cup \mathrm{B})=\mathrm{P}(\mathrm{A})+\mathrm{P}(\mathrm{B})-\mathrm{P}(\mathrm{A}) * \mathrm{P}(\mathrm{B}) \\
\mathrm{P}(\mathrm{A})=\mathrm{P}(\mathrm{CPS})=0.9 \\
\mathrm{P}(\mathrm{B})=\mathrm{P}(\mathrm{IPS})=0.825 \\
\mathrm{P}(\mathrm{A} \cup \mathrm{B})=0.9+0.825-0.9 * 0.825 \\
\mathrm{P}(\mathrm{A} \cup \mathrm{B})=0.9825
\end{gathered}
$$

Plan and goal structure reliability is evaluated wit probability $\mathrm{P}(\mathrm{GS})=1.0$

Reliability and work controlling object (OBJ) is evaluated 0.7 . Accident vector is evaluated $\mathrm{v}=0.3$.

$$
\mathrm{P}(\mathrm{OBJ})=0.7
$$

Reliability executive part of system with two executioners:

$$
\begin{gathered}
\mathrm{P}\left(\mathrm{ExPS}_{12}\right)=\mathrm{P}(\mathrm{A} \cup \mathrm{B})=\mathrm{P}(\mathrm{A})+\mathrm{P}(\mathrm{B})-\mathrm{P}(\mathrm{A}) * \mathrm{P}(\mathrm{B}) \\
\mathrm{P}\left(\mathrm{ExPS}_{1}\right)=0.9 \\
\mathrm{P}\left(\mathrm{ExPS}_{2)}=0.9\right. \\
\mathrm{P}\left(\mathrm{ExPS}_{12}\right)=0.9+0.9-0.9 * 0.9 \\
\mathrm{P}\left(\mathrm{ExPS}_{12}\right)=0.99
\end{gathered}
$$

Executive part of system work is counted with Law of total probability $[3,9,10]$ :

$$
\mathrm{P}(\text { ExPS })=\sum \mathrm{P}\left(\mathrm{H}_{\mathrm{i}}\right) * \mathrm{P}\left(\text { ExPS }_{\mathrm{i}} / \mathrm{H}_{\mathrm{i}}\right) \mathrm{i}=1,3
$$

There are three chances for realization control action, with assumption that chances have equal probability, one realization probability is $1 / 3$.

$\mathrm{P}\left(\mathrm{H}_{\mathrm{i}}\right)$ - hypothesis that controlling action realize iexecutioner

$$
\begin{gathered}
\mathrm{P}\left(\mathrm{H}_{1}\right)=\mathrm{P}\left(\mathrm{H}_{2}\right)=\mathrm{P}\left(\mathrm{H}_{12}\right)=1 / 3 \\
\mathrm{P}(\text { ExPS })=1 / 3 * 0.9+1 / 3 * 0.9+1 / 3 * 0.99 \\
\mathrm{P}(\text { ExPS })=0.93
\end{gathered}
$$

System probability in reaching demanded output or goal is: $\mathrm{p}=\mathrm{CIPS}(\mathrm{p}=0.9825) \cap \operatorname{ExPS}(\mathrm{p}=0.93) \cap \mathrm{OBJ}(\mathrm{p}=0.7) \cap \mathrm{GS}(\mathrm{p}=1.0)$

$$
\mathrm{y}=0.9825 * 0.93 * 0.7 * 1.0=0.6396 \mathrm{yd}
$$

\subsubsection{Minimum System Reliability}

Same system on Scheme 2 is looked at, controlling part system and executive part system elements (presenting union independent events) are worked with low reliability with probability 0.5 (called one man decision).

$$
\mathrm{P}(\mathrm{CPS})=\mathrm{P}\left(\operatorname{ExPS}_{1}\right)=\mathrm{P}\left(\operatorname{ExPS}_{2}\right)=1 / 2=0.50
$$

Information system reliability is shown:

$$
\begin{gathered}
\text { Pis }\left(\mathrm{p}_{\max }, \mathrm{p}_{\min }\right)=(0.95,0.70) \\
\mathrm{P}(\mathrm{IPS})=1 / 2 * 0.95+1 / 2 * 0.70 \\
\mathrm{P}(\mathrm{IPS})=0.825
\end{gathered}
$$

Information - controlling system work is presented as independent events union:

$$
\begin{gathered}
\mathrm{P}(\mathrm{A} \cup \mathrm{B})=\mathrm{P}(\mathrm{A})+\mathrm{P}(\mathrm{B})-\mathrm{P}(\mathrm{A}) * \mathrm{P}(\mathrm{B}) \\
\mathrm{P}(\mathrm{A})=\mathrm{P}(\mathrm{CPS})=0.5 \\
\mathrm{P}(\mathrm{B})=\mathrm{P}(\mathrm{IPS})=0.825
\end{gathered}
$$




$$
\begin{gathered}
\mathrm{P}(\mathrm{A} \cup \mathrm{B})=0.5+0.825-0.5 * 0.825 \\
\mathrm{P}(\mathrm{A} \cup \mathrm{B})=0.9125
\end{gathered}
$$

Plan and goal structure reliability is evaluated wit probability $\mathrm{P}(\mathrm{GS})=1.0$

Reliability and work controlling object $(\mathrm{OBJ})$ is evaluated 0.7 . Accident vector is evaluated $\mathrm{v}=0.3$.

$$
\mathrm{P}(\mathrm{OBJ})=0.7
$$

Reliability executive part of system with two executioners:

$$
\begin{gathered}
\mathrm{P}\left(\mathrm{ExPS}_{12}\right)=\mathrm{P}(\mathrm{A} \cup \mathrm{B})=\mathrm{P}(\mathrm{A})+\mathrm{P}(\mathrm{B})-\mathrm{P}(\mathrm{A}) * \mathrm{P}(\mathrm{B}) \\
\mathrm{P}\left(\mathrm{ExPS}_{1}\right)=0.5
\end{gathered}
$$

$$
\begin{gathered}
\mathrm{P}\left(\mathrm{ExPS}_{2}\right)=0.5 \\
\mathrm{P}\left(\mathrm{ExPS}_{12}\right)=0.5+0.5-0.5 * 0.5 \\
\mathrm{P}\left(\mathrm{ExPS}_{12}\right)=0.75
\end{gathered}
$$

Executive part of system work is counted with Law of total probability $[3,9,10]$ :

$\mathrm{P}($ ExPS $)=\sum \mathrm{P}\left(\mathrm{H}_{\mathrm{i}}\right) * \mathrm{P}\left(\right.$ ExPS $\left._{\mathrm{i}} / \mathrm{H}_{\mathrm{i}}\right) \mathrm{i}=1,3$

$\mathrm{P}\left(\mathrm{H}_{\mathrm{i}}\right)$ - hypothesis that ${ }^{1}$ controlling action realize $\mathrm{i}$ executioner

$\mathrm{P}\left(\mathrm{H}_{1}\right)=\mathrm{P}\left(\mathrm{H}_{2}\right)=\mathrm{P}\left(\mathrm{H}_{12}\right)=1 / 3$

$\mathrm{P}($ ExPS $)=1 / 3 * 0.5+1 / 3 * 0.5+1 / 3 * 0.75$

$\mathrm{P}(\mathrm{ExPS})=0.5833$

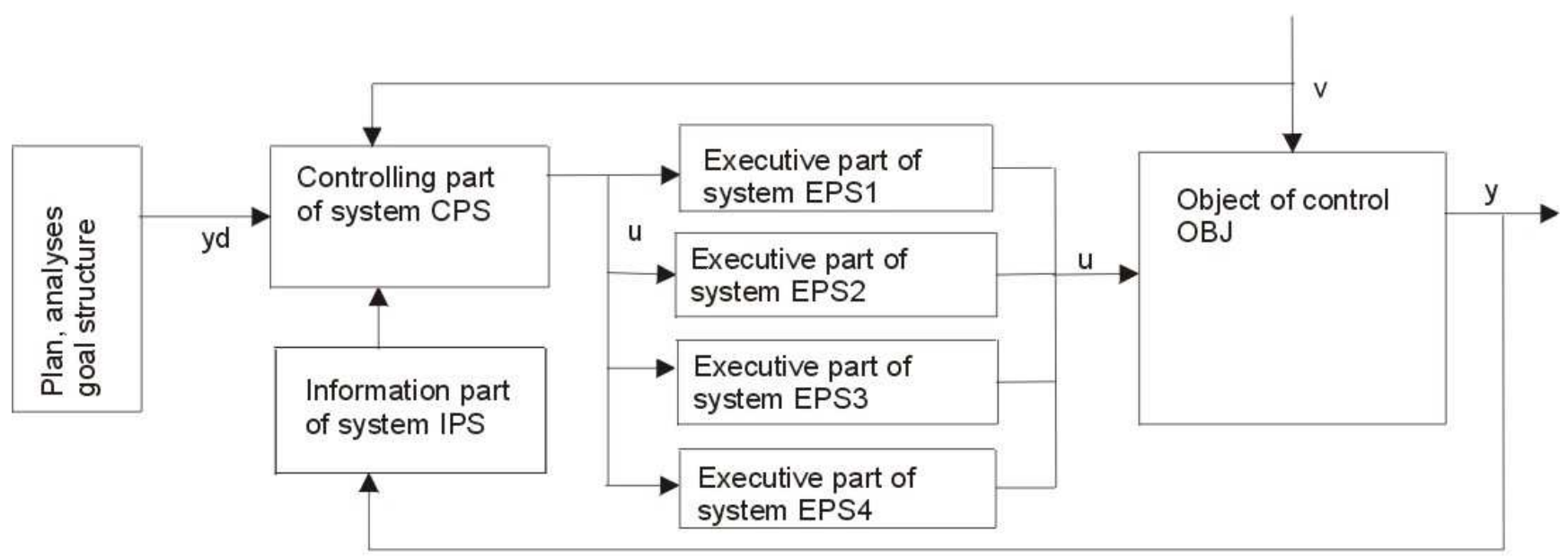

Figure 3. Structural scheme of controlled system - scheme 3 [2].

System probability in reaching demanded output or goal is:

$$
\begin{gathered}
\mathrm{p}=\operatorname{CIPS}(\mathrm{p}=0.9125) \cap \operatorname{ExPS}(\mathrm{p}=0.5833) \\
\cap \operatorname{OBJ}(\mathrm{p}=0.7) \cap \operatorname{GS}(\mathrm{p}=1.0) \\
\mathrm{y}=0.9125^{*} 0.5833 * 0.7 * 1.0=0.3726 \mathrm{yd}
\end{gathered}
$$

\subsection{System with Four Executive Parts}

Same system on Scheme 3 is looked as examples 3.1 and 3.2. The differences is that researched system have four executive parts. They are acted on same controlling instruction with possibility that each executioner can realize controlling by his own.

System reliability is counted same way, taking as events union when joined work more executioners on the same instruction is possible, or as just one execution. Executive part of system reliability is counted with Law of total probability.

$$
\mathrm{u}=\mathrm{u}(\mathrm{yd}, \mathrm{y}, \mathrm{v})
$$

\subsubsection{Maximum System Reliability}

System with four executioners work is taken into count with maximum elements reliability evaluated with probability 0.9 .

Information system reliability is shown:

$$
\begin{gathered}
\text { Pis }\left(\mathrm{p}_{\max }, \mathrm{p}_{\min }\right)=(0.95,0.70) \\
\mathrm{P}(\mathrm{IPS})=1 / 2 * 0.95+1 / 2 * 0.70 \\
\mathrm{P}(\mathrm{IPS})=0.825
\end{gathered}
$$

Information - controlling system work is presented as independent events union:

$$
\begin{gathered}
\mathrm{P}(\mathrm{A} \cup \mathrm{B})=\mathrm{P}(\mathrm{A})+\mathrm{P}(\mathrm{B})-\mathrm{P}(\mathrm{A}) * \mathrm{P}(\mathrm{B}) \\
\mathrm{P}(\mathrm{A})=\mathrm{P}(\mathrm{CPS})=0.9 \\
\mathrm{P}(\mathrm{B})=\mathrm{P}(\mathrm{IPS})=0.825 \\
\mathrm{P}(\mathrm{A} \cup \mathrm{B})=0.9+0.825-0.9 * 0.825 \\
\mathrm{P}(\mathrm{A} \cup \mathrm{B})=0.9825
\end{gathered}
$$

Plan and goal structure reliability is evaluated wit probability $\mathrm{P}(\mathrm{GS})=1.0$

Reliability and work controlling object $(\mathrm{OBJ})$ is evaluated 0.7 . Accident vector is evaluated $\mathrm{v}=0.3$.

$$
\mathrm{P}(\mathrm{OBJ})=0.7
$$

Reliability executive part of system with four executioners 


$$
\begin{gathered}
\mathrm{P}\left(\mathrm{ExPS}_{12}\right)=\mathrm{P}(\mathrm{A} \cup \mathrm{B})=\mathrm{P}(\mathrm{A})+\mathrm{P}(\mathrm{B})-\mathrm{P}(\mathrm{A} \cap \mathrm{B})=\mathrm{P}(\mathrm{A})+ \\
\mathrm{P}(\mathrm{B})-\mathrm{P}(\mathrm{A}) * \mathrm{P}(\mathrm{B}) \\
\mathrm{P}\left(\mathrm{ExPS}_{123}\right)=\mathrm{P}(\mathrm{A} \cup \mathrm{B} \cup \mathrm{C})=\mathrm{P}(\mathrm{A})+\mathrm{P}(\mathrm{B})+\mathrm{P}(\mathrm{C})-\mathrm{P}(\mathrm{A} \cap \\
\mathrm{B})-\mathrm{P}(\mathrm{A} \cap \mathrm{C})-\mathrm{P}(\mathrm{B} \cap \mathrm{C})+\mathrm{P}(\mathrm{A} \cap \mathrm{B} \cap \mathrm{C}) \\
\mathrm{P}\left(\mathrm{ExPS}_{1234}\right)=\mathrm{P}(\mathrm{A} \cup \mathrm{B} \cup \mathrm{C} \cup \mathrm{D})=\mathrm{P}(\mathrm{A})+\mathrm{P}(\mathrm{B})+\mathrm{P}(\mathrm{C})+ \\
\mathrm{P}(\mathrm{D})-\mathrm{P}(\mathrm{A} \cap \mathrm{B})-\mathrm{P}(\mathrm{A} \cap \mathrm{C})-\mathrm{P}(\mathrm{B} \cap \mathrm{C})-\mathrm{P}(\mathrm{A} \cap \mathrm{D})-\mathrm{P}(\mathrm{B} \cap \\
\mathrm{D})-\mathrm{P}(\mathrm{C} \cap \mathrm{D})+\mathrm{P}(\mathrm{A} \cap \mathrm{B} \cap \mathrm{C})+\mathrm{P}(\mathrm{A} \cap \mathrm{B} \cap \mathrm{D})+\mathrm{P}(\mathrm{A} \cap \mathrm{C} \\
\cap \mathrm{D})+\mathrm{P}(\mathrm{B} \cap \mathrm{C} \cap \mathrm{D})-\mathrm{P}(\mathrm{A} \cap \mathrm{B} \cap \mathrm{C} \cap \mathrm{D}) \\
\mathrm{P}\left(\mathrm{ExPS}_{1}\right)=0.9 \\
\mathrm{P}\left(\mathrm{ExPS}_{2}\right)=0.9 \\
\mathrm{P}\left(\mathrm{ExPS}_{3}\right)=0.9 \\
\mathrm{P}\left(\mathrm{ExPS}_{4}\right)=0.9
\end{gathered}
$$

It is possible realization assigned controlling actions with two executioners, treated as union independent events.

$$
\begin{gathered}
\mathrm{P}\left(\operatorname{ExPS}_{12}\right)=0.9+0.9-0.9 * 0.9 \\
\mathrm{P}\left(\operatorname{ExPS}_{12}\right)=0.99 \\
\mathrm{P}\left(\operatorname{ExPS}_{12}\right)=\mathrm{P}\left(\operatorname{ExPS}_{13}\right)=\mathrm{P}\left(\operatorname{ExPS}_{14}\right)=\mathrm{P}\left(\operatorname{ExPS}_{23}\right)=\mathrm{P}\left(\operatorname{ExPS}_{24}\right)= \\
\mathrm{P}\left(\mathrm{ExPS}_{34}\right)=0.99
\end{gathered}
$$

It is possible realization assigned controlling actions with three executioners, treated as union independent events.

$$
\begin{gathered}
\mathrm{P}\left(\mathrm{ExPS}_{123}\right)=0.9+0.9+0.9-0.9 * 0.9 * 3+0.9 * 0.9 * 0.9 \\
\mathrm{P}\left(\mathrm{ExPS}_{123}\right)=0.999 \\
\mathrm{P}\left(\mathrm{ExPS}_{123}\right)=\mathrm{P}\left(\mathrm{ExPS}_{124}\right)=\mathrm{P}\left(\mathrm{ExPS}_{234}\right)=\mathrm{P}\left(\mathrm{ExPS}_{134}\right)=0.999
\end{gathered}
$$

It is possible realization assigned controlling actions with four executioners, treated as union independent events.

$$
\begin{gathered}
\mathrm{P}\left(\mathrm{ExPS}_{1234}\right)=0.9+0.9+0.9+0.9-0.9 * 0.9 * 6+0.9 * 0.9 * 0.9 \\
* 4-0.9 * 0.9 * 0.9 * 0.9 \\
\mathrm{P}\left(\mathrm{ExPS}_{1234}\right)=0.9999
\end{gathered}
$$

Executive part of system work is counted with Law of total probability $[3,9,10]$ :

$$
\mathrm{P}(\mathrm{ExPS})=\sum \mathrm{P}(\mathrm{Hi}) * \mathrm{P}(\mathrm{ExPSi} / \mathrm{Hi}) \mathrm{i}=1,15
$$

There are fifteen chances to realize control action, chances have equal probability (adopted assumption), therefor one realization has probability $1 / 15$.

$\mathrm{P}(\mathrm{Hi})$ - hypothesis that controlling action realize iexecutioner

$$
\begin{gathered}
\mathrm{P}\left(\mathrm{H}_{1}\right)=\mathrm{P}\left(\mathrm{H}_{2}\right)=\mathrm{P}\left(\mathrm{H}_{3}\right)=\mathrm{P}\left(\mathrm{H}_{4}\right)= \\
\left(\mathrm{H}_{12}\right)=\mathrm{P}\left(\mathrm{H}_{13}\right)=\mathrm{P}\left(\mathrm{H}_{14}\right)=\mathrm{P}\left(\mathrm{H}_{23}\right)=\mathrm{P}\left(\mathrm{H}_{24}\right)=\mathrm{P}\left(\mathrm{H}_{34}\right)= \\
\mathrm{P}\left(\mathrm{H}_{123}\right)=\mathrm{P}\left(\mathrm{H}_{124}\right)=\mathrm{P}\left(\mathrm{H}_{234}\right)=\mathrm{P}\left(\mathrm{H}_{134}\right)=\mathrm{P}\left(\mathrm{H}_{1234}\right)=1 / 15 \\
\mathrm{P}(\mathrm{ExPS})=4 / 15 * 0.9+6 / 15 * 0.99+4 / 15 * 0.999+1 / 15 \\
* 0.9999
\end{gathered}
$$

$$
\mathrm{P}(\mathrm{ExPS})=0.9691
$$

System probability in reaching demanded output or goal is:

$$
\begin{gathered}
\mathrm{p}=\operatorname{CIPS}(\mathrm{p}=0.9825) \cap \operatorname{ExPS}(\mathrm{p}=0.9691) \\
\cap \operatorname{OOBJ}(\mathrm{p}=0.7) \cap \operatorname{GS}(\mathrm{p}=1.0) \\
\mathrm{y}=0.9825 * 0.9691 * 0.7 * 1.0=0.6665 \mathrm{yd}
\end{gathered}
$$

\subsubsection{Minimum System Reliability}

System with four executioner work is taken into count with minimum elements reliability executive part of system, as the controlling part of system with probability 0.5 .

Information system reliability is shown:

$$
\begin{aligned}
& \text { Pis }\left(\mathrm{p}_{\max }, \mathrm{p}_{\min }\right)=(0.95,0.70) \\
& \mathrm{P}(\mathrm{IPS})=1 / 2 * 0.95+1 / 2 * 0.70 \\
& \mathrm{P}(\mathrm{IPS})=0.825
\end{aligned}
$$

Information - controlling system work is presented as independent events union:

$$
\begin{gathered}
\mathrm{P}(\mathrm{A} \cup \mathrm{B})=\mathrm{P}(\mathrm{A})+\mathrm{P}(\mathrm{B})-\mathrm{P}(\mathrm{A}) * \mathrm{P}(\mathrm{B}) \\
\mathrm{P}(\mathrm{A})=\mathrm{P}(\mathrm{CPS})=0.5 \\
\mathrm{P}(\mathrm{B})=\mathrm{P}(\mathrm{IPS})=0.825 \\
\mathrm{P}(\mathrm{A} \cup \mathrm{B})=0.5+0.825-0.5 * 0.825 \\
\mathrm{P}(\mathrm{A} \cup \mathrm{B})=0.9125
\end{gathered}
$$

Plan and goal structure reliability is evaluated wit probability $\mathrm{P}(\mathrm{GS})=1.0$

Reliability and work controlled object $(\mathrm{OBJ})$ is evaluated 0.7 . Accident vector is evaluated $v=0.3$.

$$
\mathrm{P}(\mathrm{OBJ})=0.7
$$

Reliability executive part of system with four executioners:

$$
\begin{aligned}
& \mathrm{P}\left(\mathrm{ExPS}_{1}\right)=0.5 \\
& \mathrm{P}\left(\mathrm{ExPS}_{2}\right)=0.5 \\
& \mathrm{P}\left(\mathrm{ExPS}_{3}\right)=0.5 \\
& \mathrm{P}\left(\mathrm{ExPS}_{4}\right)=0.5
\end{aligned}
$$

It is possible realization assigned controlling actions with two executioners, treated as union independent events.

$$
\begin{gathered}
\mathrm{P}\left(\operatorname{ExPS}_{12}\right)=0.5+0.5-0.5 * 0.5 \\
\mathrm{P}\left(\mathrm{ExPS}_{12}\right)=0.75
\end{gathered}
$$

$$
\begin{gathered}
\mathrm{P}\left(\operatorname{ExPS}_{12}\right)=\mathrm{P}\left(\operatorname{ExPS}_{13}\right)=\mathrm{P}\left(\operatorname{ExPS}_{14}\right)=\mathrm{P}\left(\operatorname{ExPS}_{23}\right)=\mathrm{P}\left(\mathrm{ExPS}_{24}\right)= \\
\mathrm{P}\left(\mathrm{ExPS}_{34}\right)=0.75
\end{gathered}
$$

It is possible realization assigned controlling actions with three executioners, treated as union independent events.

$$
\mathrm{P}\left(\mathrm{ExPS}_{123}\right)=0.5+0.5+0.5-0.5 * 0.5 * 3+0.5 * 0.5 * 0.5
$$




$$
\begin{gathered}
\mathrm{P}\left(\operatorname{ExPS}_{123}=0.875\right. \\
\mathrm{P}\left(\mathrm{ExPS}_{123}\right)=\mathrm{P}\left(\mathrm{ExPS}_{124)}=\mathrm{P}\left(\mathrm{ExPS}_{234}\right)=\mathrm{P}\left(\mathrm{ExPS}_{134)}=0.875\right.\right.
\end{gathered}
$$

It is possible realization assigned controlling actions with four executioners, treated as union independent events.

$$
\begin{gathered}
\mathrm{P}\left(\mathrm{ExPS}_{1234}\right)=0.5+0.5+0.5+0.5-0.5 * 0.5 * 6+0.5 * 0.5 * 0.5 \\
* 4-0.5 * 0.5 * 0.5 * 0.5 \\
\mathrm{P}\left(\mathrm{ExPS}_{1234)}=0.9375\right.
\end{gathered}
$$

Executive part of system work is counted with Law of total probability $[3,9,10]$ :

$$
\mathrm{P}(\mathrm{ExPS})=\sum \mathrm{P}(\mathrm{Hi}) * \mathrm{P}(\mathrm{ExPSi} / \mathrm{Hi}) \mathrm{i}=1,15
$$

$\mathrm{P}(\mathrm{Hi})$ - hypothesis that controlling action realize iexecutioner

$$
\begin{gathered}
\mathrm{P}\left(\mathrm{H}_{1}\right)=\mathrm{P}\left(\mathrm{H}_{2}\right)=\mathrm{P}\left(\mathrm{H}_{3}\right)=\mathrm{P}\left(\mathrm{H}_{4}\right)= \\
\left(\mathrm{H}_{12}\right)=\mathrm{P}\left(\mathrm{H}_{13}\right)=\mathrm{P}\left(\mathrm{H}_{14}\right)=\mathrm{P}\left(\mathrm{H}_{23}\right)=\mathrm{P}\left(\mathrm{H}_{24}\right)=\mathrm{P}\left(\mathrm{H}_{34}\right)= \\
\mathrm{P}\left(\mathrm{H}_{123}\right)=\mathrm{P}\left(\mathrm{H}_{124}\right)=\mathrm{P}\left(\mathrm{H}_{234)}=\mathrm{P}\left(\mathrm{H}_{134}\right)=\mathrm{P}\left(\mathrm{H}_{1234}\right)=1 / 15\right. \\
\mathrm{P}(\mathrm{ExPS})=4 / 15 * 0.5+6 / 15 * 0.75+4 / 15 * 0.875+1 / 15 \\
* 0.9375
\end{gathered}
$$

$$
\mathrm{P}(\mathrm{ExPS})=0.7292
$$

System probability in reaching demanded output or goal is:

$$
\begin{gathered}
\mathrm{p}=\operatorname{CIPS}(\mathrm{p}=0.9125) \cap \operatorname{ExPS}(\mathrm{p}=0.7292) \\
\cap \operatorname{OBJ}(\mathrm{p}=0.7) \cap \operatorname{GS}(\mathrm{p}=1.0) \\
\mathrm{y}=0.9125^{*} 0.7292 * 0.7 * 1.0=0.4658 \text { yd }
\end{gathered}
$$

From shown calculation is concluded that reliability system increase with rise of executioner number in controlling.

\section{Conclusion}

Presented analysis has intended to show reliability systems with high human resources representation, system with balanced proportion human and automatic machines work about $50 \%$ to $50 \%$ [11-15].

In production goodness where human labor is high qualified, automatic machine use is not justified, because creative manual work is underestimated, having in mind employment and production quality. Higher participation human labor in production goodness is justified.

Involving higher technical percentage in production accident vector became smaller about $\mathrm{v} \sim 20 \%$ or $\mathrm{v} \sim 10 \%$.

Presented analysis is involved new concept in researching cybernetics systems.

\section{References}

[1] V. Vujičić and B. Petrović, Cybernetics (Kibernetika), Naučna knjiga, Beograd 1979.

[2] B. Đorđević, Water resources systems (Vodoprivredni sistemi), Naučna knjiga, Beograd 1990.

[3] J. Mališić and V. Jevremović, Statistical analyze and stochastic processes (Statistička analiza i slučajni procesi), Naučna knjiga, Beograd 1991.

[4] N. Wiener, Cybernetics: or control and communication in the animal and the machine, Cambridge, Massachusets: MIT Press, 1948.

[5] L. T. Kuzin, Osnovy kibernetiki, "Energija”, Moskva 1973.

[6] J. Mališić, Stochastic processes theory and applications (Slučajni procesi teorija i primena), Građevinska knjiga, Beograd 1989.

[7] S. Opricović, Multicriterion systems optimization in civil engineering (Višekriterijumska optimizacija sistema u građevinarstvu), Građevinski fakultet Univerziteta u Beogradu, 1998.

[8] Z. Ivković and D. Banjević, Probability and mathematical statistics (Verovatnoća i matematička statistika), Naučna knjiga, Beograd 1981.

[9] J. Mališić, Problems collection of probability theory with applications (Zbirka zadataka iz teorije verovatnoće sa primenama), Građevinska knjiga, Beograd 1982.

[10] P. M. Vasić, Exercises and problems of probability theory (Zadaci i problemi iz teorije verovatnoće), Građevinska knjiga, Beograd 1982.

[11] D. Dimitrijević, Computational programme (Računarsko programiranje), Naučna knjiga, Beograd 1986.

[12] D. Dimitrijević and Lj. Savić, Computational programme problems collection (Računarsko programiranje zbirka rešenih zadataka), Naučna knjiga, Beograd 1986.

[13] B. Vasić and V. Popović, Managing methods of engineering (Inženjerske metode menadžmenta), Institut za istraživanja i projektovanja u privredi, Beograd 2007.

[14] Đ. Marković, J. Plavšić, M. Stanić and G. Sekulić, Nonparametric estimation of density function in hidrology (Neparametarske funkcije raspodele u hidrologiji), Vodoprivreda Vol. 43 (2011) 249-251, Beograd.

[15] B. Đorđević and T. Dašić, Method for determinig the enviromental flow downstrem od the dams and water intakes (Određivanje potrebnih protoka nizvodno od brana i rečnih vodozahvata), Vodoprivreda Vol. 43 (2011) 252-254, Beograd. 